\title{
HOMOLOGY TANGENT BUNDLES AND UNIVERSAL BUNDLES $^{1}$
}

\author{
DANIEL HENRY GOTTLIEB
}

\begin{abstract}
We find results about the evaluation map from the group of homeomorphisms of a closed manifold $M$ and also about fibre bundles where $M$ is the fibre. These facts follow from the observation that the homology tangent bundle is induced from a universal bundle pair.
\end{abstract}

1. Introduction. The object of this paper is to prove the following theorem:

THEOREM 12. Let $G$ be any group of homeomorphisms acting on a closed oriented topological manifold $M$ and let $\omega: G \rightarrow M$ be the evaluation map at the base point *. Then $\chi(M) \omega *: \tilde{H}^{*}(M ; R) \rightarrow \tilde{H}^{*}(G ; R)$ is trivial where $R$ is any ring of coefficients with a unit and $\chi(M)$ is the Euler-Poincaré number of $M$.

Note that the theorem applies to the coset mapping $\rho: G \rightarrow G / H$.

The proof of Theorem 12 makes use of the following observation: Let $G$ be the path-connected component of the group of homeomorphisms of any topological manifold $M$ and let $H$ be the isotropy subgroup at $*$. Then we have the fibre bundle $M \rightarrow{ }^{i} B_{I I} \rightarrow B_{G}$.

THEOREM 5. The inclusion $i$ induces the homology tangent bundle from a "universal" fibre pair over $B_{H}$.

We use a theorem of R. F. Brown to obtain Theorem 12 from Theorem 5. Along the way we obtain a topological version of a theorem of Borel [2].

THEOREM 9. Let $M \rightarrow E \rightarrow{ }^{\pi} B$ be any fibre bundle with an orientation preserving structural group, where $M$ is a closed, orientable, topological manifold. Let $p$ be a prime such that $\chi(M) \not \equiv 0 \bmod p$. Then $\pi^{*}: H^{*}\left(B ; Z_{p}\right) \rightarrow$ $H^{*}\left(E ; Z_{p}\right)$ is injective.

Received by the editors October 14, 1971 and, in revised form, January 7, 1972.

AMS 1970 subject classifications. Primary 55E99, 55F05, 57A65, 55F10.

Key rords and phrases. Homology tangent bundle, classifying bundle, Euler class, characteristic classes, topological manifolds, actions.

1 The preparation of this paper was sponsored in part by the National Science Foundation, Grant number GP 29465X1. 
2. Preliminaries. Let $G$ be a topological group and let $H$ be a closed subgroup. Let $E$ be a space on which $G$ operates on the right such that the projections $E \rightarrow E / G$ and $E \rightarrow E / H$ are principal fibre bundles with fibres $G$ and $H$ respectively. Also, we have the fibre bundle $\rho: E / H \rightarrow E / G$ with fibre $G / H$.

Let $G$ operate on the left of a space $F$. Then, as usual, $E \times{ }_{G} F$ will denote the quotient space of $E \times F$ under the equivalence relation $(e, x) \sim$ $\left(e g, g^{-1} x\right)$. The equivalence class of $(e, x)$ will be denoted by $\langle e, x\rangle$. Thus $\langle e, x\rangle$ is a point of $E \times_{G} F$ and $\langle e, x\rangle=\left\langle e g, g^{-1} x\right\rangle$. Similarly, we shall denote points of $E / H$ by $\langle e\rangle_{H}$ where $e \in E$ and points of $E / G$ will be denoted $\langle e\rangle_{G}$.

The map $p: E \times_{G} F \rightarrow E / G$ given by $p(\langle e, x\rangle)=\langle e\rangle_{G}$ is the projection of a fibre bundle with fibre $F$ and group $G$.

Also, we need the concept of bundles induced by a map. Let $E \rightarrow^{p} B$ be a bundle and $X \rightarrow^{f} B$ be a map. The induced bundle $f^{*}(X) \rightarrow^{p(f)} X$ is a bundle with the same fibre and $f^{*}(X)$ is the subspace of $E \times X$ given by points of the form $(e, x)$ such that $p(e)=f(x)$. We shall denote points in $f^{*}(X)$ by $(e, x)$. Then the projection map $p(f)$ is given by $(e, x) \mapsto x$.

We wish to consider the square of the bundle $E / H \rightarrow^{\rho} E / G$. This is the bundle $G / H \rightarrow \rho^{*}(E / H) \rightarrow E / H$. The points of $\rho^{*}(E / H)$ have the form $\left(\langle e\rangle_{H},\left\langle e^{\prime}\right\rangle_{H}\right)$ where $\langle e\rangle_{G}=\left\langle e^{\prime}\right\rangle_{G}$. Note there is a unique $g \in G$ such that $e^{\prime} \cdot g=e$.

There is another bundle with fibre $G / H$ over $E / H$. This is $G / H \rightarrow$ $E \times_{H} G / H \rightarrow E / H$. These two bundles are equivalent. We record some standard facts below for the reader's convenience:

LEMMA 1. The two bundles $\rho^{*}(E / H) \rightarrow E / H$ and $E \times_{H} G / H \rightarrow E / H$ are equivalent. The bundle equivalence is given by the map $\rho^{*}(E / H) \rightarrow^{\alpha}$ $E \times_{H} G / H$ which sends $\left(\langle e\rangle_{H},\left\langle e^{\prime}\right\rangle_{H}\right) \mapsto\left\langle e, g^{-1} H\right\rangle$ where $g$ is defined by the equation $e^{\prime} \cdot g=e$.

LEMMA 2. There exists a cross-section $s: E / H \rightarrow \rho^{*}(E / H)$ to the fibration $\rho^{*}(E / H) \rightarrow E / H$ given by $\langle e\rangle_{H^{\prime}} \mapsto\left(\langle e\rangle_{H},\langle e\rangle_{H}\right)$. The fibration restricts over the fibre $G / H$ of $E / H \rightarrow^{\rho} E / G$ to projection on the second factor $G / H \times G / H \rightarrow G / H$. Then the cross-section $s$ restricted to the fibre $G / H$ is the diagonal $G / H \rightarrow{ }^{\Delta}$ $G / H \times G / H$. We may also regard $s$ as the cross-section $E / H \rightarrow E \times{ }_{H} G / H$ given by $\langle e\rangle_{H} \mapsto\langle e, H\rangle$.

LEMMA 3. There is a commutative diagram of fibre bundles

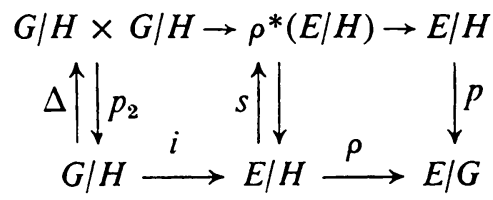


LEMMA 4. There is a bundle equivalence

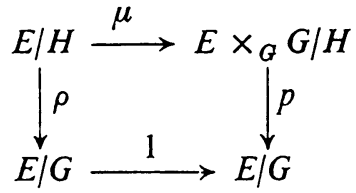

where $\mu\left(\langle e\rangle_{H}\right)=\langle e, H\rangle$.

3. The homology tangent bundle. In this section we shall always assume that $G / H$ is a topological manifold without boundary, which we shall also denote by $M$. Also, $G$ will be a group of homeomorphisms of $M$ onto itself, endowed with the compact open topology. Then $H$ will be a space of homeomorphisms of $M$ which leave the base point $* \in M$ fixed, i.e. the isotropy subgroup. We shall always take $E$ to be contractible. Thus we have $B_{G}=E / G$ and $B_{H}=E / H$.

Now observe that $H$ may be regarded as a group of homeomorphisms of $M-*$. Consider the universal principal bundle $H \rightarrow E \rightarrow B_{H}$. By regarding $H$ as operating on $M$, we obtain the associated bundle $M \rightarrow E \times_{H} M \rightarrow B_{H}$. By regarding $H$ as operating on $M-*$ we obtain the associated bundle $(M-*) \rightarrow E \times_{H}(M-*) \rightarrow B_{H}$.

Observe that $E \times_{H}(M-*)$ is contained as a subspace in $E \times_{H} M$. In fact, using the cross-section $s$ of Lemma 2, we have $E \times{ }_{H}(M-*)=$ $\left(E \times_{H} M\right)-s\left(B_{H}\right)$. The pair $\left(E \times_{H} M, E \times_{H}(M-*)\right)$ is a fibre-bundle pair in the sense of Fadell [6]. See also [10, p. 256]. The fibre is $(M, M-*)$ and the base is $B_{H}$.

By Lemma 1, we may view the fibre pair from a different point. Recall the fibre bundle $M \rightarrow^{i} B_{H} \rightarrow^{\rho} B_{G}$. Then $E \times_{H} M$ is just $\rho^{*}\left(B_{H}\right)$, and $E \times{ }_{H}(M-*)$ is $\rho^{*}\left(B_{H}\right)-s\left(B_{H}\right)$. Thus it is easy to see, using Lemma 3, that the fibre bundle $M \times M \rightarrow^{D_{2}} M$ given by projection on the second factor is induced by $i: M \rightarrow B_{H}$ from the bundle $\rho^{*}\left(B_{H}\right) \rightarrow B_{H}$. Also, the bundle $M \times M-\Delta \rightarrow^{p_{2}} M$ given by projection on the second factor is induced by $i: M \rightarrow B_{H}$ from the bundle $\rho^{*}\left(B_{H}\right)-s\left(B_{H}\right) \rightarrow B_{H}$. We formalize this in the following theorem.

THEOREM 5. The bundle pair $(M \times M, M \times M-\Delta) \rightarrow^{D_{2}} M$ with fibre $(M, M-*)$ is induced from the bundle pair $\left(\rho^{*}\left(B_{H}\right), \rho^{*}\left(B_{H}\right)-s\left(B_{H}\right)\right) \rightarrow B_{H}$ by the inclusion map $i: M \rightarrow B_{I I}$.

Now let $\left(E^{\prime}, E_{0}^{\prime}\right)$ be a fibre pair with fibre $\left(F, F_{0}\right)$ and base space $B$. Then $\pi_{1}(B, *)$ operates on $H_{*}\left(F, F_{0} ; Z\right)$. The fibre pair $\left(E^{\prime}, E_{0}^{\prime}\right)$ is said to be orientable if $\pi_{1}(B, *)$ operates trivially on $H_{*}\left(F, F_{0} ; Z\right)$. If $\pi_{1}(B, *)$ operates trivially on $H_{*}\left(F, F_{0} ; Z_{2}\right)$, the fibre pair is called $Z_{2}$-orientable. 
LEMMA 6. (a) The homology tangent bundle

$$
(M, M-*) \rightarrow(M \times M, M \times M-\Delta) \stackrel{p_{2}}{\longrightarrow} M
$$

is $Z_{2}$-orientable. If $M$ is orientable, then the homology tangent bundle is orientable.

(b) Let $M$ be orientable and let $G$ be a group of homeomorphisms of $M$ which preserve orientation. Then the fibre pair

$$
(M, M-*) \rightarrow\left(\rho^{*}\left(B_{H}\right), \rho^{*}\left(B_{H}\right)-s\left(B_{H}\right)\right) \rightarrow B_{H}
$$

is orientable.

(c) For any $M$ and $G$, the above fibre pair is $Z_{2}$-orientable.

Proof. (a) was proved by Fadell in [6].

(b) Let $\alpha \in \pi_{1}\left(B_{H}, *\right)$. We shall show that $\alpha$ acts trivially on $H_{n}(M, M-*)$. There exists an isotopy $f_{t}: M \rightarrow B_{H}$ such that:

(1) each $f_{t}$ is a homeomorphism of $M$ onto a fibre of $B_{H} \rightarrow{ }^{p} B_{G}$.

(2) $f_{0}$ is the identity map of $M$ regarded as the fibre over $p(*)$,

(3) the trace of the isotopy (i.e. the path $\sigma: t \mapsto f_{t}(*)$ ) is a closed path representing $\alpha$.

Now we define an isotopy $g_{t}: M \rightarrow \rho^{*} B_{H}$ by setting $g_{t}(m)=\left(f_{t}(m), f_{t}(*)\right) \in$ $\rho^{*} B_{I H}$. Note that $g_{t}$ is an isotopy of the pairs $(M, M-*) \rightarrow$ $\left(\rho^{*}\left(B_{H}\right), \rho^{*}\left(B_{H}\right)-s\left(B_{H}\right)\right)$.

Now $g_{0}$ is the identity on the fibre $(M, M-*)$ over $* \in B_{H}$. On the other hand $g_{1}(m)=\left(f_{1}(m), *\right) \in(M, M-*)$. Since $f_{1}$ preserves orientation on $M$ by hypotheses, we see that $g_{1}:(M, M-*) \rightarrow(M, M-*)$ must induce the identity homomorphism on $H_{n}(M, M-*)$. Thus $\alpha$ acts trivially on $H_{n}(M, M-*)$, which was to be shown.

(c) is obvious since $H_{n}\left(M, M-* ; Z_{2}\right) \cong Z_{2}$.

Let $\left(E^{\prime}, E_{0}^{\prime}\right) \rightarrow B$ be an orientable fibre bundle pair with fibre $(M, M-*)$. Since $H^{*}(M, M-*)=H^{*}\left(R^{m}, R^{m}-0\right)$, there exists a "Thom isomorphism" $\phi: H^{i}(B) \cong H^{i+n}\left(E^{\prime}, E_{0}^{\prime}\right)$ which is natural with respect to mappings of fibre bundle pairs. Then $\phi(1) \in H^{n}\left(E^{\prime}, E_{0} ; R\right)$, where $R$ is a ring of coefficients, is the "Thom class". Characteristic classes (Fadell [6]) are defined from the Thom class in the obvious way. For example, $\phi^{-1}(\phi(1) \cup \phi(1))$ is the Euler class.

The characteristic classes of $M$ are defined to be the characteristic classes of the homology tangent bundle $(M, M \times M-\Delta)$.

COROLLARY 7. If $k \in H^{i}(M ; R)$ is a characteristic class of $M$, then $k$ is in the image of $i^{*}: H^{i}\left(B_{H} ; R\right) \rightarrow H^{i}(M ; R)$.

Now we assume that $M$ is a closed orientable $n$-manifold. Then R. F. Brown [4] says the Euler class of $M$ is equal to $\chi(M) \mu$ where $\mu \in H^{n}(M ; R)$ 
is the fundamental class of the manifold and $\chi(M)$ is the Euler-Poincare number of $M$. If $\varepsilon \in H^{n}\left(B_{H} ; R\right)$ is the Euler class for the fibre pair $\left(\rho^{*}\left(B_{H}\right), \rho^{*}\left(B_{H}\right)-s\left(B_{H}\right)\right) \rightarrow B_{H}$, then by Theorem 5 we have $i^{*}(\varepsilon)=$ $\chi(M) \mu$.

Corollary 8. $i^{*}(\varepsilon)=\chi(M) \mu$.

\section{Proof of Theorem 9.}

THEOREM 9. Let $M \rightarrow E \rightarrow^{p} B$ be a fibre bundle with orientation preserving structural group and let $M$ be a closed, orientable topological manifold. If $\chi(M) \not \equiv 0(\bmod p)$, then $p^{*}$ is injective. In addition, the theorem is still true when $p=2$ with no orientability condition on $M$ or on the fibre bundle.

Proof. We know that the Euler class of $M$ is $\chi(M) \mu \in H^{n}(M ; Z)$, where $\mu$ is a generator, by a theorem of R. F. Brown [4]. Let $\chi(M) \mu$ also denote the element in $Z_{p}$ cohomology. If $\chi(M) \not \equiv 0(\bmod p)$, then

$$
\chi(M) \mu \neq 0 \in H^{n}\left(M ; Z_{p}\right) .
$$

Consider the fibration $M \rightarrow{ }^{i} B_{H} \rightarrow{ }^{p} B_{G}$. By Corollary $8, \chi(M) \mu$ is in the image of $i^{*}: H^{n}\left(B_{H} ; Z_{p}\right) \rightarrow H^{n}\left(M ; Z_{p}\right)$. (Note that in order to apply Corollary 8 we need that Lemma 6 be valid. But Lemma 6(b) requires that $G$ be orientation preserving.) For $Z_{2}$ coefficients we apply (c). By naturality, $\chi(M) \mu$ is in the image of $i^{*}$ for the fibration $M \rightarrow^{i} E \rightarrow^{p} B$.

Lemma 3.1 of [2] (or see Theorem 14.5 of [3]) says the following: Suppose $F \rightarrow^{i} E \rightarrow^{p} B$ is a fibration with an $n$-dimensional fibre $F$ such that $\pi_{1}(B)$ acts trivially on $H^{n}\left(F ; Z_{p}\right)$. If $i^{*}: H^{n}\left(E ; Z_{p}\right) \rightarrow H^{n}\left(F ; Z_{p}\right)$ is nonzero, then $p^{*}$ is injective.

In the case at hand, $\pi_{1}(B)$ operates trivially on $H^{n}\left(M ; Z_{p}\right)$ since $\pi_{1}(B)$ operates trivially on the image of $i^{*}$ (which is onto $H^{n}\left(M ; Z_{p}\right)$ ).

REMARK. In a forthcoming paper, we shall remove the hypothesis that $M$ is orientable.

5. Actions and characteristic classes. In this section we shall prove that a characteristic class of a topological manifold $M$ is "inert" under the action $\hat{\omega}: G \times M \rightarrow M$. We say $k \in H^{*}(M ; R)$ is inert under $\hat{\omega}$ if $\hat{\omega}^{*}(k)=$ $1 \times k$, where $R$ is any ring of coefficients. One consequence of the inertness of characteristic classes is that $\chi(M) \omega^{*}$ is trivial where $\omega: G \rightarrow M$ is evaluation at a base point $*$.

We begin by studying the action $\hat{\omega}: G \times(G / H) \rightarrow G / H$ given by $\hat{\omega}\left(g, g^{\prime} H\right)=g g^{\prime} H$. This action fits inside the following commutative diagram. 


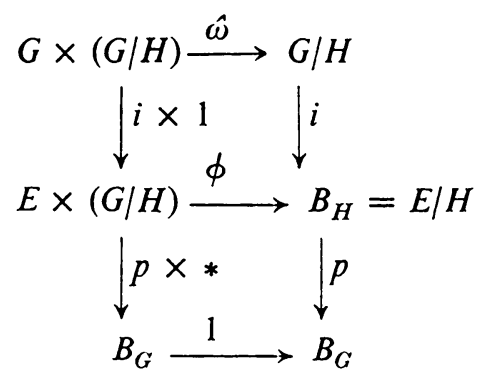

where $\phi$ is defined by $\phi(e, g H)=\langle e g\rangle_{H}$ and $i: G \rightarrow E$ is given by $i(g)=g$ and $i: G / H \rightarrow E / H$ is given by $i(g H)=\langle g\rangle_{H}$. Observe that $\phi$ is well defined and continuous and that $\left({ }^{*}\right)$ in fact commutes.

Since $E$ is contractible, the commutativity of $\left(^{*}\right)$ tells us that $i \circ \hat{\omega}$ is homotopic to $G \times(G / H) \rightarrow{ }^{\operatorname{proj}} G / H \rightarrow{ }^{i} B_{H}$. This proves the following theorem.

THEOREM 10. Any $k$ which is in the image of $i^{*}: H^{*}\left(B_{I} ; R\right) \rightarrow$ $H^{*}(G / H ; R)$ is inert under the action $\hat{\omega}$. In particular, characteristic classes of topological manifolds are inert under $\hat{\omega}$.

REMARK. The inertness of characteristic classes of differentiable manifolds under differentiable actions will be shown in [9] (i.e. Stiefel-Whitney classes and Pontrjagin class are inert under differentiable actions).

The usefulness of the concept of inertness comes from the following lemma. Let $\omega: G \rightarrow M$ be given by $\omega=\hat{\omega}(\cdot, *)$ for base point $* \in M$.

Lemma 11. Suppose that $u$ and $v$ are positive dimensional cohomology elements of a space $M$, and suppose that $v$ is inert under some action $\hat{\omega}$. Then $u \cup v=0$ implies $\omega^{*}(u) \times v=0$.

Proof. We have

$$
\begin{aligned}
0 & =\hat{\omega}^{*}(u \cup v)=\hat{\omega}^{*}(u) \cup \hat{\omega}^{*}(v) \\
& =\left(\omega^{*}(u) \times 1+\sum a_{i} \times b_{i}+\sum c_{i} * d_{i}\right) \cup 1 \times v \\
& =\omega^{*}(u) \times k+\sum a_{i} \times\left(b_{i} \cup k\right)+\sum\left(c_{i} * d_{i}\right) \cup(1 \times v) .
\end{aligned}
$$

Here the $a_{i}$ and $c_{i} \in H^{*}(M ; R)$ are positive dimensional and $b_{i}$ and $d_{i} \in$ $H^{*}(G ; R)$ and $\times$ is the cohomology cross product and $*$ is the torsion product coming from the Künneth formula. It is easy to see that no term in the expansion has the right dimensions to cancel $\omega^{*}(u) \times k$. Hence $\omega^{*}(u) \times k=0$.

The above lemma, with the aid of Corollary 8 gives us the main result. 
THEOREM 12. Let $M$ be a closed orientable topological manifold and let $G$ be a group of homeomorphisms acting on $M$ by the action $\hat{\omega}: G \times M \rightarrow M$. Let $\omega: G \rightarrow M$ be the evaluation map at the base point *. Then $\chi(M) \omega^{*}$ : $\tilde{H}^{*}(M ; R) \rightarrow \tilde{H}^{*}(G ; R)$ is trivial where $R$ is any coefficient ring with unit.

Proof. By Corollary $8, i^{*}(\varepsilon)=\chi(M) \mu$. So $\chi(M) \mu$ is inert by Theorem 10. (We let $\chi(M) \mu$ also stand for the image of $\chi(M) \mu$ in cohomology with coefficients in $R$.) Since $\mu$ is the top dimensional class, $\mu \cup v=0$ for any $v \in \tilde{H}^{*}(M ; R)$. Thus, by Lemma 11 ,

$$
0=\omega^{*}(v) \times \chi(M) \mu=\chi(M) \omega^{*}(v) \times \mu .
$$

So $\chi(M) \omega^{*}(v)$ must equal zero, thus proving the theorem.

REMARK. If $R=Z_{2}$, the orientability requirement may be dropped.

\section{BIBLIOGRAPHY}

1. Guy Allaud, On the classification of fibre spaces, Math. Z. 92 (1966), 110-125. MR 32 \#6462.

2. Armand Borel, Sur la torsion des groupes de Lie, J. Math. Pures Appl. (9) 35 (1956), 127-139. MR 17. 1107.

3. - Topics in the homology theory of fibre bundles, Lecture Notes in Math., no. 36, Springer-Verlag, Berlin and New York, 1967. MR 36 \#4559.

4. Robert F. Brown, On the Lefschetz number and the Euler class, Trans. Amer. Math. Soc. 118 (1965), 174-179. MR 30 \#3481.

5. Albrecht Dold, Halbexakte Homotopiefunktoren, Lecture Notes in Math., no. 12, Springer-Verlag, 1966 (see p. 16.8). MR 33 \#6622.

6. Edward Fadell, Generalized normal bundles for locally-flat imbeddings, Trans. Amer. Math. Soc. 114 (1965), 488-513. MR 31 \#4037.

7. D. H. Gottlieb, On fibre spaces and the evaluation map, Ann. of Math. (2) 87 (1968), 42-55; Corrigendum, ibid. (2) 91 (1970), 640-642. MR 36 \#4560; MR 41 \#7684.

8. _ Evaluation subgroups of homotopy groups, Amer. J. Math. 91 (1969), 729 756.

9. - Applications of bundle map theory, Trans. Amer. Math. Soc. (to appear).

10. Edwin H. Spanier, Algebraic topology, McGraw-Hill, New York, 1966. MR 35 \#1007.

Department of Mathematics, Purdue University, West lafayette, Indiana 47907 